\title{
Practical TDMA for Datacenter Ethernet
}

\author{
Bhanu C. Vattikonda, George Porter, Amin Vahdat, Alex C. Snoeren \\ Department of Computer Science and Engineering \\ University of California, San Diego \\ \{bvattikonda, gmporter, vahdat, snoeren\}@cs.ucsd.edu
}

\begin{abstract}
Cloud computing is placing increasingly stringent demands on datacenter networks. Applications like MapReduce and Hadoop demand high bisection bandwidth to support their all-to-all shuffle communication phases. Conversely, Web services often rely on deep chains of relatively lightweight RPCs. While HPC vendors market niche hardware solutions, current approaches to providing high-bandwidth and lowlatency communication in the datacenter exhibit significant inefficiencies on commodity Ethernet hardware.

We propose addressing these challenges by leveraging the tightly coupled nature of the datacenter environment to apply time-division multiple access (TDMA). We design and implement a TDMA MAC layer for commodity Ethernet hardware that allows end hosts to dispense with TCP's reliability and congestion control. We evaluate the practicality of our approach and find that TDMA slots as short as 100s of microseconds are possible. We show that partitioning link bandwidth and switch buffer space to flows in a TDMA fashion can result in higher bandwidth for MapReduce shuffle workloads, lower latency for RPC workloads in the presence of background traffic, and more efficient operation in highly dynamic and hybrid optical/electrical networks.
\end{abstract}

Categories and Subject Descriptors C.2.1 [ComputerCommunication Networks]: Network Architecture and Design-Network communications.

General Terms Performance, Measurement, Experimentation.

Keywords Datacenter, TDMA, Ethernet.

Permission to make digital or hard copies of all or part of this work for personal or classroom use is granted without fee provided that copies are not made or distributed for profit or commercial advantage and that copies bear this notice and the full citation on the first page. To copy otherwise, to republish, to post on servers or to redistribute to lists, requires prior specific permission and/or a fee.

EuroSys'12, April 10-13, 2012, Bern, Switzerland.

Copyright (c) 2012 ACM 978-1-4503-1223-3/12/04 . . \$10.00

\section{Introduction}

The size, scale, and ubiquity of datacenter applications are growing at a rapid pace, placing increasingly stringent demands on the underlying network layer. Datacenter networks have unique requirements and characteristics compared to wide-area or enterprise environments: Today's datacenter network architects must balance applications' demands for low one-way latencies (sometimes measured in $10 \mathrm{~s}$ of microseconds or less), high bandwidth utilization-i.e., 10 Gbps at the top-of-rack switch and increasingly in end hosts-and congestion-free operation to avoid unanticipated queuing delays. This goal is complicated by the dynamic nature of the traffic patterns and even topology within some datacenters. A flow's path, and the available bandwidth along that path, can change on very fine timescales [4].

The applications that must be supported in datacenter environments can have drastically varying requirements. On one hand, data-intensive scalable computing (DISC) systems like MapReduce [9], Hadoop, and TritonSort [21] can place significant demands on a network's capacity. DISC deployments are often bottlenecked by their all-to-all shuffle phases, in which large amounts of state must be transferred from each node to every other node. On the other hand, modern Web services are increasingly structured as a set of hierarchical components that must pass a series of small, inter-dependent RPCs between them in order to construct a response to incoming requests [18]. The overall throughput of these so-called Partition/Aggregate workloads [2] is frequently gated by the latency of the slowest constituent RPC. Similarly, structured stores like BigTable [6] or their front-ends (e.g., Memcached) require highly parallel access to a large number of content nodes to persist state across a number of machines, or to reconstruct state that is distributed through the datacenter. In these latter cases, low-latency access between clients and their servers is critical for good application performance.

While hardware vendors have long offered boutique link layers to address extreme application demands, the cost advantages of Ethernet continue to win out in the vast majority of deployments. Moreover, Ethernet is increasingly capable, pushing toward 40- and even 100-Gbps 
link bandwidths. Switch vendors have also begun to offer lower-latency switches supporting cut-through forwarding along a single network hop. Recent proposals for datacenter design have suggested leveraging this increasing hardware performance-even including optical interconnectsthrough fine-grained, dynamic path selection $[10,26]$. In these environments, TCP transport becomes a major barrier to low-latency, high-throughput intercommunication. Indeed, Facebook reportedly eschews TCP in favor of a custom UDP transport layer [22], and the RAMCloud prototype dispenses with Ethernet entirely (in favor of Infiniband) due to its poor end-to-end latency [16].

We argue that these datacenter communication patterns look less like the traditional wide-area workloads TCP was designed to handle, and instead resemble a much more tightly coupled communication network: the back-plane of a large supercomputer. We seek to provide support for high-bandwidth and low latency-specifically all-to-all bulk transfers and scatter-gather type RPCs - in this much more controlled environment, where one can forego the distributed nature of TCP's control loop. In order to dispense with TCP, however, one must either replace its reliability and congestion control functionality, or remove the need for it. Here, we seek to eliminate the potential for congestion, and, therefore, queuing delay and packet loss. To do so, we impose a time-division multiple access (TDMA) MAC layer on a commodity Ethernet network that ensures end hosts have exclusive access to the path they are assigned at any point in time.

In our approach, we deploy a logically centralized link scheduler that allocates links exclusively to individual sender-receiver pairs on a time-shared basis. In this way, link bandwidth and switch buffer space is exclusively assigned to a particular flow, ensuring that in-network queuing and congestion is minimized or, ideally, eliminated. As such, our approach is a good fit for cut-through switching fabrics, which only work with minimal buffering, as well as future generations of hybrid datacenter optical circuit switches [10, 24, 26] which have no buffering. Our technique works with commodity Ethernet NICs and switching hardware. It does not require modifications to the network switches, and only modest software changes to end hosts. Because we do not require time synchronization among the end hosts, our design has the potential to scale across multiple racks and even entire datacenters. Instead, our centralized controller explicitly schedules end host NIC transmissions through the standardized IEEE $802.3 \mathrm{x}$ and $802.1 \mathrm{Qbb}$ protocols. A small change to these protocols could allow our approach to scale to an even larger number of end hosts.

In this paper, we evaluate the practicality of implementing TDMA on commodity datacenter hardware. The contributions of our work include 1) a TDMA-based Ethernet MAC protocol that ensures fine-grained and exclusive access to links and buffers along datacenter network paths, 2) a reduction in the completion times of bulk all-to-all transfers by approximately $15 \%$ compared to TCP, 3 ) a $3 \times$ reduction in latency for RPC-like traffic, and 4) increased TCP throughput in dynamic network and traffic environments.

\section{Related work}

We are far from the first to suggest providing stronger guarantees on Ethernet. There have been a variety of proposals to adapt Ethernet for use in industrial automation as a replacement for traditional fieldbus technologies. These efforts are far too vast to survey here ${ }^{1}$; we simply observe that they are driven by the need to provide real-time guarantees and expect to be deployed in tightly time-synchronized environments that employ real-time operating systems. For example, FTT-Ethernet [19] and RTL-TEP [1] both extend real-time operating systems to build TDMA schedules in an Ethernet environment. RTL-TEP further leverages timetriggered Ethernet (TT-Ethernet), a protocol that has gone through a variety of incarnations. Modern implementations of both TT-Ethernet [13] and FTT-Ethernet [23] require modified switching hardware. In contrast to these real-time Ethernet (RTE) proposals, we do not require the use of realtime operating systems or modified hardware, nor do we presume tight time synchronization.

The IETF developed Integrated Services [5] to provide guaranteed bandwidth to individual flows, as well as controlled load for queue-sensitive applications. IntServ relies on a per-connection, end-host-originated reservation packet, or RSVP packet [30], to signal end-host requirements, and support from the switches to manage their buffers accordingly. Our work differs in that end hosts signal their demand and receive buffer capacity to a logically centralized controller, which explicitly schedules end-host NICs on a per-flow basis, leaving the network switches unmodified.

Our observation that the traffic patterns seen in datacenter networks differ greatly from wide-area traffic is well known, and many researchers have attempted to improve TCP to better support this new environment. One problem that has received a great deal of attention is incast. Incast occurs when switch buffers overflow in time-spans too quick for TCP to react to, and several proposals have been made to avoid incast $[2,7,20,25,28]$. TDMA, on the other hand, can be used to address a spectrum of potentially complimentary issues. In particular, end hosts might still choose to employ a modified TCP during their assigned time slots. While we have not yet explored these enhanced TCPs, we show in Section 6.4 that our TDMA layer can improve the performance of regular TCP in certain, nonincast scenarios.

One limitation of a TDMA MAC is that the benefits can only be enjoyed when all of the end hosts respect the schedule. Hence, datacenter operators may not want to deploy

\footnotetext{
${ }^{1}$ http://www.real-time-ethernet.de/ provides a nice compendium of many of them.
} 
TDMA network-wide. Several proposals have been made for ways of carving up the network into different virtual networks, each with their own properties, protocols, and behaviors. Notable examples of this approach to partitioning include VINI [3], OpenFlow [17], and Onix [14]. Webb et al. [27] introduce topology switching to allow applications to deploy individual routing tasks at small time scales. This work complements ours, as it enables datacenter operators to employ TDMA on only a portion of their network.

\section{Motivation and challenges}

A primary contribution of this work is evaluating the feasibility of deploying a TDMA MAC layer over commodity Ethernet switches and end hosts. In this section we describe how a TDMA MAC layer could improve the performance of applications in today's datacenters and leverage future technologies like hybrid packet-circuit switched networks.

\subsection{Motivation}

The TCP transport protocol has adapted to decades of changes in underlying network technologies, from widearea fiber optics, to satellite links, to the mobile Web, and to consumer broadband. However, in certain environments, such as sensor networks, alternative transports have emerged to better suit the particular characteristics of these networks. Already the datacenter is becoming such a network.

\subsubsection{Supporting high-performance applications}

TCP was initially applied to problems of moving data from one network to another, connecting clients to servers, or in some cases servers to each other. Contrast that with MapReduce and Hadoop deployments [29] and Memcached installations (e.g., at Facebook), which provide a datacenterwide distributed memory for multiple applications. The traffic patterns of these distributed applications look less like traditional TCP traffic, and increasingly resemble a much more tightly coupled communication network. Recent experiences with the incast problem show that the parallel nature of scatter-gather type problems (e.g., distributed search index queries), leads to packet loss in the network. [2, $7,20,25,28]$ When a single query is farmed out to a large set of servers, which all respond within a short time period (often within microseconds of each other), those packets overflow in-network switch buffers before TCP can detect and respond to this temporary congestion. Here a more proactive, rather than reactive, approach to managing innetwork switch buffers and end hosts would alleviate this problem.

One critical aspect of gather-scatter workloads is that they are typically characterized by a large number of peer nodes. In a large Memcached scenario, parallel requests are sent to each of the server nodes, which return partial results, which the client aggregates together to obtain the final result returned to the user. The latency imposed by these lookups can easily be dominated by the variance of response time seen by the sub-requests. So while a service might be built for an average response time of 10 milliseconds, if half of the requests finish in $5 \mathrm{~ms}$, and the other half finish in $15 \mathrm{~ms}$, the net result is a $15-\mathrm{ms}$ response time.

\subsubsection{Supporting dynamic network topologies}

Datacenters increasingly employ new and custom topologies to support dynamic traffic patterns. We see the adoption of several new technologies as a challenge for current transport protocols. As bandwidth requirements increase, relying on multiple network paths has become a common way of increasing network capacity. Commodity switches now support hashing traffic at a flow-level across multiple parallel data paths. A key way to provide network operators with more flexibility in allocating traffic to links is supporting finer-grained allocation of flows to links. This promises to improve link (and network) utilization. At the same time, a single TCP connection migrating from one link to another might experience a rapidly changing set of network conditions.

The demand for fine-grained control led to the development of software-defined network controllers, including OpenFlow [17]. Through OpenFlow, novel network designs can be built within a logically centralized network controller, leaving data path forwarding to the switches and routers spread throughout the network. As the latency for reconfiguring the network controller shrinks, network paths might be reconfigured on very small timescales. This will pose a challenge to TCP, since its round-trip time and available throughput estimates might change due to policy changes in the network, rather than just due to physical link failures and other more infrequent events.

Another scenario in which flow paths change rapidly arises due to network designs that propose to include optical circuit switches within datacenters. The advantages of optical switches include lower energy, lower price and lower cabling complexity as compared to electrical options. These benefits currently come at the cost of higher switching times, but they are rapidly decreasing. Technologies as DLPbased wavelength selective switches can be reconfigured in $10 \mathrm{~s}$ to $100 \mathrm{~s}$ of microseconds [15], at which point it will no longer be possible to choose circuit configurations by reacting to network observations [4]. Instead, the set of switch configurations will have to be programmed in advance for a period of time. In this model, if the end hosts and/or switches can be informed of the switch schedule, they can coordinate the transmission of packets to make use of the circuit when it becomes available to them. Our TDMA mechanism would naturally enable this type of microsecondgranularity interconnect architecture.

\subsection{Challenges}

As a starting point, we assume that a datacenter operator either deploys TDMA throughout their entire network, or that 
they rely on OpenFlow or some other isolation technology to carve out a portion of their network to devote to TDMA. Within the portion of the network dedicated to TDMA, we rely upon a centralized controller to compute and distribute a schedule that specifies an assignment of slots to individual end hosts. Each slot represents permission for a host to send to a particular destination: when a host is assigned to a slot, it can communicate with that destination at full link capacity and be guaranteed not to experience any cross traffic, either on the links or at the switches.

The feasibility of our proposed approach depends on how effectively one can schedule Ethernet transmissions. Clearly the overhead of per-packet polling is too high, so end hosts must be in charge of scheduling individual packet transmissions. It is an open question, however, what else should be managed by the end hosts, versus what canor needs to be-controlled in a centralized fashion. The answer depends upon the following features of commodity hardware:

1. The (in)ability of end-host clocks to stay synchronized;

2. The effectiveness with which an external entity can signal end hosts to begin or cease transmitting or, alternatively, the precision with which end hosts can keep time; and

3. The variability in packet propagation times as they traverse the network, including multiple switches.

Here, we set out to answer these questions empirically by evaluating the behavior of Ethernet devices in our testbed. (Results with other Ethernet NICs from different manufacturers are similar.) The results show that end-host clocks very quickly go out of sync; hence, we cannot rely entirely on end hosts to schedule Ethernet transmissions. On the other hand, we find that existing Ethernet signaling mechanisms provide an effective means for a centralized fabric manager to control end hosts' Ethernet transmissions in order to enforce a TDMA schedule.

\subsubsection{End-host clock skew}

The most basic of all questions revolves around how time synchronization should be established. In particular, a straightforward approach would synchronize end-host clocks at coarse timescales (e.g., through NTP), and rely upon the end hosts themselves to manage slot timing. In this model, the only centralized task would be to periodically broadcast the schedule of slots; end hosts would send data at the appropriate times.

The feasibility of such an approach hinges on how well different machines' clocks are able to stay in sync. Previous studies in the enterprise and wide area have found significant inter-host skew [8, 12], but one might conjecture that the shared power and thermal context of a datacenter reduces the sources of variance. We measure the drift between machines in our testbed (described in Section 6) by having the nodes each send packets to the same destination at pre-determined

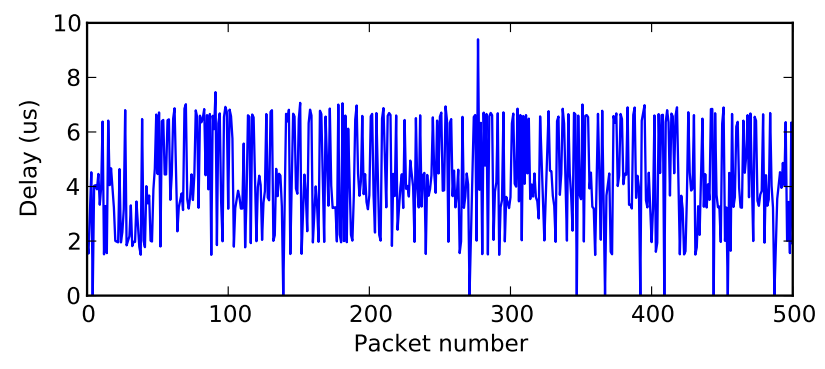

Figure 1. Delay in responding to $802.3 x$ pause frames when transmitting 64-byte packets.

intervals, and examine the differences in arrival times of the subsequent packets. At the beginning of the experiment the destination broadcasts a "sync" packet to all the senders to initialize their clocks to within a few microseconds.

We find that the individual nodes in our testbed rapidly drift apart from each other, and, in as little as 20 seconds, some of the senders are as much as $2 \mathrm{~ms}$ out of sync; i.e., in just one second senders can be out of sync by $100 \mu$ s. Given that a minimum-sized (64-byte) frame takes only $0.05 \mu \mathrm{s}$ to transmit at $10 \mathrm{Gbps}$, it becomes clear that end hosts need to be resynchronized on the order of every few milliseconds to prevent packet collisions. Conversely, it appears possible for end hosts to operate independently for 100s of microseconds without ill effect from clock skew. Hence, we consider a design where an external entity starts and stops transmissions on that timescale, but allows the end hosts to manage individual packet transmissions.

\subsubsection{Pause frame handling}

Of course, it is difficult for application-level software on today's end hosts to react to network packets in less than a few 10 s of microseconds [16], so signaling every $100 \mu \mathrm{s}$ seems impractical — at least at the application level.

Luckily, the Ethernet specification includes a host of signaling mechanisms that can be leveraged to control the end hosts' access to the Ethernet channel, many offered under the banner of datacenter bridging (DCB) [11]. One of the oldest, the $802.3 \mathrm{x}$ flow-control protocol, has long been implemented by Ethernet NICs. $802.3 x$ was originally designed to enable flow control at layer 2: When the receiver detects that it is becoming overloaded, it sends a link-local pause frame to the sender, with a configurable pause time payload. This pause time is a 16-bit value that represents the number of 512-bit times that the sender should pause for, and during that time, no traffic will be sent by the sender. On a 10-Gbps link, a single bit-time is about $51 \mathrm{ns,} \mathrm{therefore} \mathrm{the}$ maximum pause time that can be expressed is about $3.4 \mathrm{~ms}$.

To understand the granularity with which we can control end-host traffic, we measure how quickly an Ethernet sender responds to $802.3 x$ pause frames. We set up an experiment in which a single sender sends minimum-size (64-byte) packets to a receiver as fast as possible. The receiver periodically 

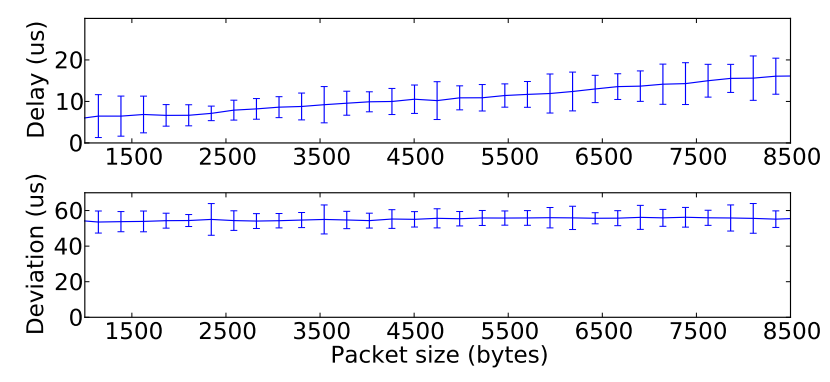

Figure 2. Average delay before pausing and deviation from the requested 3.4-ms interval as a function of the size of packets being sent by sender.

sends pause frames to the sender for the full pause amount ( $3.4 \mathrm{~ms}$ ), and we measure the lag between when the pause frame is sent and when the packets stop arriving. As shown in Figure 1, The pause frame mechanism engages quite rapidly in our NICs, generally reacting between 2-6 $\mu$ s after the frame was transmitted. Of course, the absolute delay is less important than the variance, which is similarly small.

The $802.3 \mathrm{x}$ specification requires that a sender defer subsequent transmissions upon receipt of a pause frame, but does not insist that it abort any current frame transmission. Hence, the delay before pausing increases linearly with the packet size at the sender as shown in the top portion of Figure 2. It is not clear, however, how well commodity NICs respect the requested pause time. The bottom portion of Figure 2 shows the average deviation in microseconds from the requested interval $(3.4 \mathrm{~ms}$ in this experiment). While constant with respect to the sender's packet size (implying the NIC properly accounts for the time spent finishing the transmission), it is significant. Hence, in our design we do not rely on the end host to "time out." Instead, we send a subsequent pause frame to explicitly resume transmission as explained in the next section.

\subsubsection{Synchronized pause frame reception}

Enforcing TDMA slots with $802.3 x$ pause frames simplifies the design of the end hosts, which can now become entirely reactive. However, such a design hinges on our ability to transmit (receive) pause frames to (at) the end hosts simultaneously. In particular, to prevent end hosts from sending during another's slot, the difference in receive (and processing) time for pause frames must be small across a wide set of nodes. The previous experiments show that the delay variation at an individual host is small (on the order of $5 \mu \mathrm{s}$ or less), so the remaining question is how tightly can one synchronize the delivery of pause frames to a large number of end hosts.

Because our end host clocks are not synchronized with enough precision to make this measurement directly, we instead indirectly measure the level of synchronization by measuring the difference in arrival times of a pair of control packets at 24 distinct receivers. In this experiment, we con-

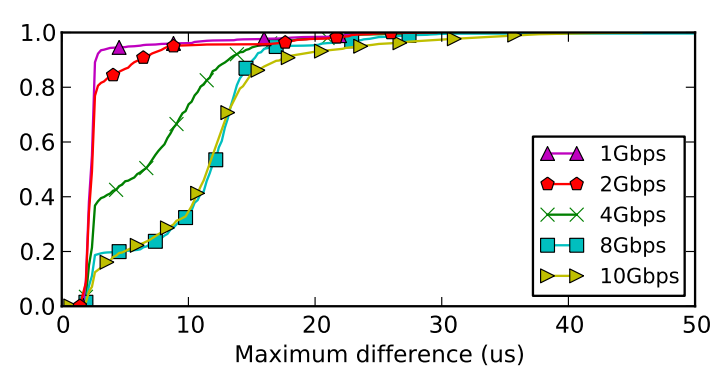

Figure 3. CDF of difference of inter-packet arrival of two pause packets for various host sending rates.

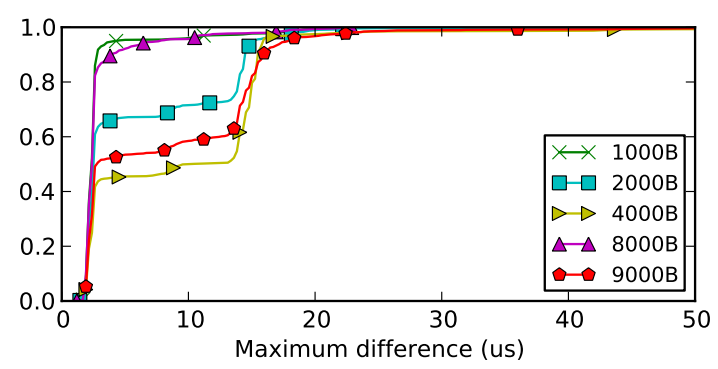

Figure 4. CDF of difference of inter-packet arrival of the control packets for various data packet sizes.

nect all the hosts to a single switch and a control host sends control packets serially to all of the other hosts. ${ }^{2}$ To simulate the TDMA scenario where these pause packets represent the end of a slot, we have the hosts generate traffic of varying intensity to other end hosts. By comparing the difference in perceived gap between the pair of control packets at each end host, we factor out any systemic propagation delay.

The cumulative distribution function (CDF) of the interpacket arrival times of the control packets at the end hosts for various packet sizes and sending rates of the traffic being generated are shown in Figures 3 and 4, respectively. The inter-host variation is on the order of $10-15 \mu$ s for the vast majority of packet pairs, and rarely more than $20 \mu \mathrm{s}$. These values guide our selection of guard times as described in Section 4.

Of course, one might worry that the story changes as the topology gets more complicated. We repeat the experiments with a multi-hop topology consisting of a single root switch and three leaf switches. Hosts are spread across the leaf switches, resulting in a 3-hop path between sets of end hosts. The results are almost indistinguishable from the single-hop case, giving us confidence that we can control a reasonable number of end hosts (at least a few racks' worth) in a centralized fashion-at least when the controller has symmetric connectivity to all end hosts, as would be the case if it was attached to the core of a hierarchical switching

\footnotetext{
${ }^{2}$ While it is not clear that $802.3 x$ pause frames were intended to be forwarded, in our experience switches do so when appropriately addressed.
} 

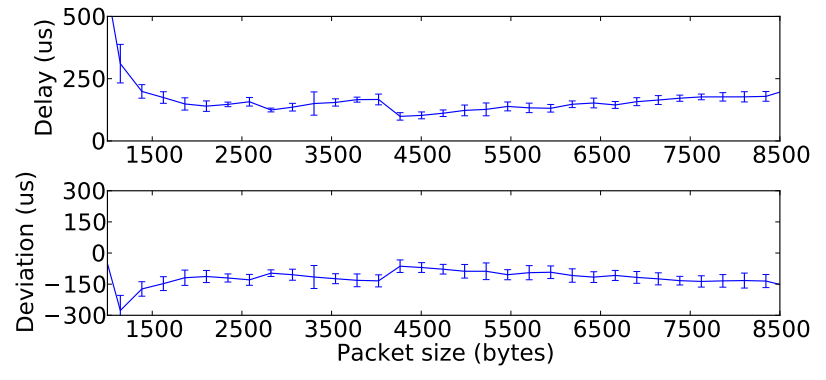

Figure 5. Average delay before pausing and deviation from the requested interval using PFC code as a function of the size of packets being sent.

topology or we attach the controller to each of the leaf switches.

\subsubsection{Traffic differentiation}

While $802.3 x$ pause frames are supported by most Ethernet cards, they unfortunately pause all traffic on the sender, making them less useful for our purposes, as we wish to selectively pause (and unpause) flows targeting various destinations. For traffic patterns in which a sender concurrently sends to multiple destinations, we require a more expressive pause mechanism that can pause at a flow-level granularity. One candidate within the DCB suite is the 802.1Qbb priority-based flow control (PFC) format, which supports 8 traffic classes. By sending an 802.1Qbb PFC pause frame, arbitrary subsets of these traffic classes can be paused. While 802.1 Qbb flow control is supported by a wide range of modern Ethernet products (e.g., Cisco and Juniper equipment), the 10-Gbps NICs in our testbed do not naively support PFC frames. Hence, we implement support in software.

While we have no reason to believe native $802.1 \mathrm{Qbb}$ PFC processing will differ substantially from $802.3 x$ pause frame handling when implemented on the NIC, our userlevel software implementation is substantially more coarse grained. To lower the latency we rely on the kernel-bypass network interface provided by our Myricom hardware. Figure 5 shows the response time of PFC frames as seen by the application-layer (c.f. the $802.3 x$ performance in Figure 2). Here we see that the average delay in responding to PFC frames is an order of magnitude higher than before, at approximately 100-200 $\mu$ s for most packet sizes. Fortunately, the variation in this delay remains low. Hence, we can still use a centralized controller to enforce slot times; the end hosts' slots will just systematically lag the controller.

\subsubsection{Alternative signaling mechanisms}

While our design leverages the performance and pervasiveness of Ethernet Priority Flow Control, there are a variety of other signaling mechanisms that might be employed to control end host transmissions within any given datacenter, some more portable than others. As long as the operating system or hypervisor can enqueue packets for different destinations into distinct transmit queues (e.g., by employing Linux NetFilter rules that create a queue per IP destination), a NIC could use its own proprietary mechanisms to communicate with the controller to determine when to drain each queue. For example, we are exploring modifying the firmware in our Myricom testbed hardware to generate and respond to a custom pause-frame format which would provide hardware support for a much larger set of traffic classes than 802.1Qbb.

\section{Design}

We now discuss the design of our proposed TDMA system. Due to the fundamental challenges involved in tightly timesynchronizing end hosts as discussed in Section 3, we choose to centralize the control at a network-wide fabric manager that signals the end hosts when it is time for them to send. For their part, end hosts simply send traffic (at line rate) to the indicated (set of) destination(s) when signaled by the controller, and remain quiet at all other times. We do not modify the network switches in any way. The fabric manager is responsible for learning about demand, scheduling flows, and notifying end hosts when and to whom to send data.

At a high-level, the fabric manager leads the network through a sequence of rounds, where each round consists of the following logical steps.

1. Hosts communicate their demand to the fabric manager on a per-destination basis.

2. The fabric manager aggregates these individual reports into a network-wide picture of total system demand for the upcoming round.

3. The fabric manager computes a communication pattern for the next round, dividing the round into fixedsize slots, during which each link is occupied by noncompeting flows (i.e., no link is oversubscribed). We call this assignment of source/destination flows to slots a schedule.

4. At the start of a round, the fabric manager informs each of the end hosts of (their portion of) the schedule for the round, and causes them to stop sending traffic, in effect muting the hosts.

5. At the start of each TDMA slot-as determined by the clock at the fabric manager-the fabric manager sends an "unpause" packet to each host that is scheduled to transmit in that slot. This packet encodes the destination of flows that should be transmitted in the slot. At the end of the slot, the fabric manager sends a "pause" packet to the host indicating that it should stop sending packets.

For efficiency reasons, several of these steps are pipelined and run in parallel with previous rounds. We now describe some of the components of our design. 


\subsection{Demand estimation}

In our proposed design, each round consists of a set of fixedsized slots, each assigned to a sender-destination pair. The optimal size of the slots depends on the aggregate network demand for that round-slots should be as large as possible without leaving any dead time-and the number of slots assigned to each host depends on the demand at each end host. Estimating future demand is obviously a challenging task. An optimal solution would instrument applications to report demand, in much the same way as applications are occasionally annotated with prefetching hints. Such an expectation seems impractical, however.

We seek to simplify the prediction task by keeping round size small, so each end host needs only report demand over a short time period, e.g., $10 \mathrm{~ms}$. At that timescale, our experience shows that it is possible to extract the necessary demand information from the operating system itself rather than the applications - at least for large transfers. For example, demand can be collected by analyzing the size of socket buffers, an approach also employed by other datacenter networking proposals like c-Through [26].

It is much more challenging, however, to estimate the demand for short flows in an application-transparent fashion. If multiple short flows make up part of a larger session, it may be possible to predict demand for the session in aggregate. For cases where demand estimation is fundamentally challenging - namely short flows to unpredictable destinations - it may instead be better to handle them outside of the TDMA process. For example, one might employ a network virtualization technology to reserve some amount of bandwidth for short flows that would not be subject to TDMA. Because short flows require only a small share of the fabric bandwidth, the impact on overall efficiency would be limited. One could then mark the short flows with special tags (QoS bits, VLAN tags, etc.) and handle their forwarding differently. We have not yet implemented such a facility in our prototype.

For TDMA traffic, demand can be signaled out of band, or a (very short) slot can be scheduled in each round to allow the fabric manager to collect demand from each host. Our current prototype uses explicit out of band demand signaling; we defer a more thorough exploration of demand estimation and communication to future work.

\subsection{Flow control}

Demand estimation is only half the story, however. An important responsibility of a network transport is ensuring that a sender does not overrun a receiver with more data than it can handle. This process is called flow control. Because nodes send traffic to their assigned destinations during the appropriate slot, it is important that those sending hosts are assured that the destinations are prepared to receive that data. In TCP this is done in-band by indicating the size of the receive buffer in ACK packets. However, in our approach we do not presume that there are packets to be sent directly from the receiver to the sender. Instead, we leverage the demand estimation subsystem described above. In particular, demand reports also include the sizes of receive buffers at each end host in addition to send buffers. In this way, the fabric manager has all the information it needs to avoid scheduling slots that would cause the receiver to drop incoming data due to a buffer overflow. While the buffer sizes will vary during the course of a round-resulting in potentially suboptimal scheduling - the schedule will never assign a slot where there is insufficient demand or receive buffer. We limit the potential inefficiency resulting from our periodic buffer updates by keeping the rounds as short as practical.

\subsection{Scheduling}

In a traditional switched Ethernet network, end hosts opportunistically send data when it becomes available, and indirectly coordinate amongst themselves by probing the properties of the source-destination path to detect contention for resources. For example, TCP uses packet drops and increases in the network round-trip time (resulting from queuing at switches) as indications of congestion. The collection of end hosts then attempt to coordinate to arrive at an efficient allocation of network resources. In our centralized model, the scheduler has all the information it needs to compute an optimal schedule. What aspects it should optimize for-e.g., throughput, fairness, latency, etc.-depends greatly on the requirements of the applications being supported. Indeed, we expect that a real deployment would likely seek to optimize for different metrics as circumstances vary.

Hence, we do not advocate for a particular scheduling algorithm in this work; we limit our focus to making it practical to carry out a given TDMA schedule on commodity Ethernet. Our initial design computes weighted round-robin schedules, where each host is assigned a slot in a fixed order before being assigned a new slot in the round. The delay between slots for any particular sender is therefore bounded.

A particular challenge occurs when multiple senders have data to send to the same destination, but none of them have sufficient data to fill an entire slot themselves. Alternatives include using a smaller slot size, or combining multiple senders in one slot. Slot sizes are bounded below by practical constraints. Due to the bursty nature of (even paced) transmissions on commodity NICs, however, combining multiple senders into one slot can potentially oversubscribe links at small timescales, which requires buffering at the switch. Again, we defer this complexity to future work and focus on schedules that assign slots exclusively to individual senders.

This issue becomes even more complex in networks with mixed host link rates, such as those with some hosts that have gigabit Ethernet NICs and others with 10-Gbps NICs. In such a network, a fixed-size slot assigned to a 10-Gbps transmitter will exceed the capacity of a 1-Gbps receiver to receive traffic in the same slot. One alternative is to share the slot at the transmitter among destinations (for example, ten 
1-Gbps receivers). Another is to buffer traffic at the switch. We could leverage our flow control mechanism to ensure a switch was prepared to buffer a slot's worth of traffic at $10 \mathrm{Gbps}$ for an outgoing port, and then schedule that port to drain the queue for the next 9 slots. We have not yet incorporated either of these possibilities into our prototype.

\subsection{Scale}

Perhaps the most daunting challenge facing a centralized design comes from the need to ensure that pause packets from the controller arrive in close proximity at the nodes, especially when the network can have an arbitrary topology. In the ideal case, the fabric manager is connected to the same switch as the hosts it controls, but such a topology obviously constrains the size of the deployment to the number of hosts that can be connected to a single switch. While that suffices for, say, a single rack, multi-rack deployments would likely require the system to function with end hosts that are connected to disparate switches.

While the function of the scheduler is logically centralized, the actual implementation can of course be physically distributed. Hence, one approach is to send pause frames not from one fabric manager, but instead from multiple slave controllers that are located close to the end hosts they control, but are themselves synchronized through additional means such as GPS-enabled NICs.

We have not yet implemented such a hierarchical design in our prototype. Instead, we scale by employing a single physical controller with multiple NICs that are connected directly to distinct edge switches. Using separate threads to send pause frames from each NIC attached to the controller, we control hosts connected to each edge switch in a manner which resembles separate slave controllers with synchronized clocks. So far, we have tested up to 24 hosts per switch; using our current topology and 8 NICs in a single, centralized controller, the approach would scale to 384 hosts. Multiple such controllers which have hardware synchronize clocks would need to be deployed to achieve scalability to thousands of end hosts. So long as each switch is at the same distance to the end hosts being controlled, this approach can work for arbitrary topologies.

In a large-scale installation, these two techniques can be combined. I.e., multiple physical controllers can coordinate to drive a large number of hosts, where each controller is directly connected to multiple switches. The scale of this approach is bounded by the number of NICs a controller can hold, the number of ports on each switch, and the ability to tightly time synchronize each slave controller-although the latter is easily done by connecting all the slave controllers to a control switch and triggering the transmission of pause frames using a link-layer broadcast frame.

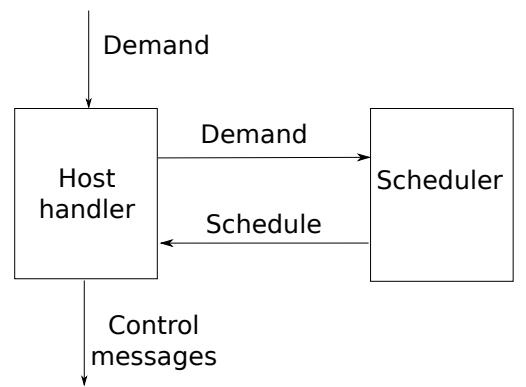

Figure 6. The main components of the fabric manager

\section{Implementation}

Our prototype implementation consists of a centralized, multi-core fabric manager that communicates with application-level TDMA agents at the end hosts that monitor and communicate demand as well as schedule transmission. Slots are coordinated through 802.1 Qbb PFC pause frames.

\subsection{PFC message format}

Unlike the original use case for Ethernet PFC, our system does not use these frames to pause individual flows, but rather the converse: we pause all flows except the one(s) that are assigned to a particular slot. Unfortunately, the priority flow control format currently being defined in the IEEE 802.1Qbb group allows for only 8 different classes of flows. To support our TDMA-based scheduling, one has to either classify all the flows from a host into these 8 classes or perform dynamic re-mapping of flows to classes within a round. While either solution is workable, in the interest of expediency (since we implement PFC support in software anyway) we simply extend the PFC format to support a larger number of classes.

In our experiments that use fewer than 8 hosts, we use the unmodified frame format; for larger deployments we modify the PFC format to support a 11-bit class field, rather than the 3-bit field dictated by the $802.1 \mathrm{Qbb}$ specification. We remark, however, that since the PFC frames are only acted upon by their destination, the fabric manager can reuse PFC classes across different nodes, as long as those classes are not reused on the same link. Thus, the pause frame does not need enough classes to support all the flows in the datacenter, but rather only the flows on a single link.

\subsection{Fabric manager}

The fabric manager has two components as shown in Figure 6. One component is the Host Handler and other component is Scheduler. All the tasks of interacting with the hosts are done by the host handler while the actual scheduling is done by the Scheduler component. The Scheduler is a pluggable module depending on the underlying network topology and the desired scheduling algorithm.

The fabric manager needs to be aware of both the sending demand from each end host to calculate slot assignments, as 
well as the receiving capacity to support flow control. The role of the Host Handler is to receive the above mentioned demand and capacity information from the end hosts and share it with the Scheduler. End hosts send their demand to the Host Handler out-of-band in our implementation, and that demand is used by the Scheduler for the next round of slot assignments. The slot assignments are sent back to the end hosts by the Host Handler. During each round the Host Handler sends extended PFC packet frames to each of the end hosts to instigate the start and stop of each TDMA slot.

\subsubsection{Host Handler}

The Host Handler is implemented in two threads, each pinned to their own core so as to reduce processing latency. The first thread handles receiving demand and capacity information from the hosts, and the second is responsible for sending control packets to the end hosts. The actual demand analysis is performed by the scheduler, described next.

Once the new schedule is available, a control thread sends the pause frames to the end hosts to control the destination to which each host sends data. The control packet destined for each host specifies the class of traffic which the host can send (the unpaused class). In our testbed, the fabric manager is connected to each edge switch to reduce the variance in sending the PFC packet frames to the end hosts. When the pause frames are scheduled to be sent to the end hosts, the controller sends the pause frames to the end hosts one after the other. The pause frames are sent to all the hosts under a switch before moving on to the next switch. The order of the switches and the order of hosts under a switch changes in a round robin fashion.

\subsubsection{Scheduler}

The scheduler identifies the flows that are going to be scheduled in each slot. It does this with the goal of achieving high overall bandwidth and fairness among hosts with the constraint that no two source-destination flows use the same link at the same time. This ensures that each sender has unrestricted access to its own network path for the duration of the slot. The scheduler updates the demand state information whenever it receives demand information from the Host Handler and periodically computes the schedule and forwards it back to the Host Handler. The scheduler is pluggable, supporting different implementations. It is invoked for each round, parameterized with the current demand and capacity information obtained during the previous set of rounds.

In our implementation we employ a round-robin scheduler that leverages some simplifying assumptions about common network topologies (namely that they are trees) in order to compute the schedule for the next round during the current round. The computational complexity of this task scales as a function of both the size of the network and the communication pattern between the hosts. At some point, the time required to collect demand and compute the next schedule may become a limiting factor for round size. Developing a scheduler that can compute a schedule for arbitrary topologies in an efficient manner remains an open problem.

\subsection{End hosts}

As discussed previously, the NICs in our experimental testbed do not naively support PFC, and thus we handle these control packets at user-level. We rely on a kernel-bypass, user-level NIC firmware to reduce latency on processing PFC packets by eliminating the kernel overhead. We are able to read packets off the wire and process them in user space in about $5 \mu \mathrm{s}$.

\subsubsection{End-host controller}

We separate the implementation of the controller into distinct processes for control, sending and receiving. This is based on our observation that the responsiveness of the control system to control packets has greater variance if the sending and receiving is done in the same process using separate threads. This was true even if we pinned the threads to separate cores. Thus, our implementation has the separate processes implementing our service communicate through shared memory. The control packets arriving at the end hosts specify which class of traffic (e.g., source-destination pair) should be sent during a slot. Hosts receive the flowto-priority class mapping out of band. The control process handles the PFC message and informs the sending process of the destination to which data can be sent.

\subsubsection{Sender and receiver}

The sending process sends data to the appropriate destination during the assigned slot. If this host is not scheduled in a particular slot then the sending process remains quiescent. To simplify sending applications, we present an API similar to TCP in that it copies data from the application into a send buffer. This buffer is drained when the sending process gets a slot assignment and sent to the destination as raw Ethernet frames. We use indexed queues so that performing these data transfers are constant-time operations. The receiving process receives the incoming data and copies the data into receive buffers. The application then reads the data from the receive buffer pool through a corresponding API. As before, these are constant time operations.

\subsection{Guard times}

As detailed in Section 3.2, end host timing and pause frame processing is far from perfect. Moreover, at high overall network utilization, small variances in packet arrival times can cause some in-network switch buffering, resulting in inband control packets getting delayed, which further reduces the precision of our signaling protocol. We mitigate this phenomenon by introducing guard times between slots. These are "safety buffers" that ensure that small discrepancies in synchronization do not cause slot boundaries to be violated. 
We have empirically determined (based largely on the experiments in Section 3.2) on our testbed hardware that that a guard time of $15 \mu \mathrm{s}$ is sufficient to separate slots. This guard time is independent of the slot time and depends on the variance in the control packet processing time at the hosts and the in-network buffer lengths. The cost of the guard time is of course best amortized by introducing large slot times; however, there is a trade-off between the slot time and how well dynamic traffic changes are supported.

We implement guard times by first sending a pause frame to stop all the flows in the network followed $15 \mu$ s later by the PFC packet frame that unpauses the appropriate traffic class at each host for the next slot. The $15-\mu$ s pause in the system is enough to absorb variances in end host transmission timing and drain any in-network buffers; hence, our PFC frames reach the hosts with greater precision.

\section{Evaluation}

We now evaluate our TDMA-based system in several scenarios on a modest-sized testbed consisting of quad-core Xeon servers running Linux 2.6.32.8 outfitted with two 10GE Myricom NICs. The hosts are connected together using Cisco Nexus 5000 series switches in varying topologies as described below. In summary, our prototype TDMA scheme 1) achieves about $15 \%$ shorter finish times than TCP for all-to-all transfers in different topologies, 2) can achieve $3 \times$ lower RTTs for small flows (e.g., Partition/Aggregate workloads) in the presence of long data transfers, 3 ) achieves higher throughput for transfer patterns where lack of coordination between the flows dramatically hurts TCP performance, and 4) can improve TCP performance in rapidly changing network topologies.

\subsection{All-to-all transfer}

First, we consider the time it takes to do an all-to-all transfer (i.e., the MapReduce shuffle) in different topologies. Due do the lack of coordination between TCP flows without TDMA, some flows finish ahead of others. This can be problematic in situations when progress cannot be made until all the transfers are complete.

\subsubsection{Non-blocking topology}

In our first all-to-all transfer experiment, we connect 24 hosts to the same switch and transfer 10 GB to each other. Ideally, such a transfer would finish in 184 seconds. The top portion of Figure 7 shows the performance of a TCP all-toall transfer in this set up. The figure plots the progress of each flow with time. We see that some flows finish early at the expense of other flows, and, hence, the overall transfer takes substantially more time than necessary, completing in 225 seconds.

Contrast that with bottom portion of Figure 7, which similarly plots the progress of the hosts while running our TDMA prototype. Due to the limitation of our end
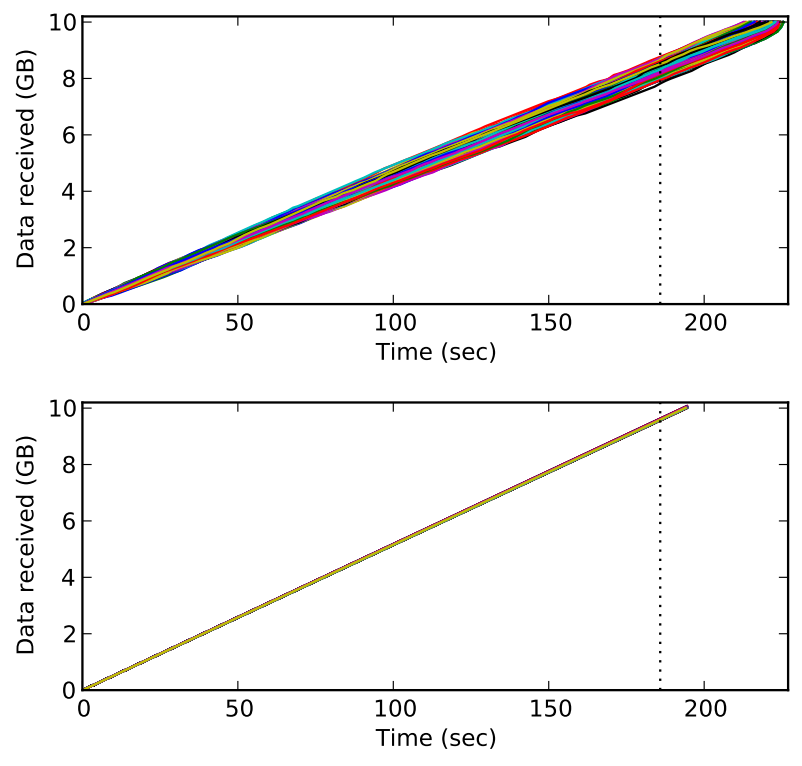

Figure 7. 10-GB all-to-all transfer between 24 hosts connected to the same switch. TCP over regular Ethernet takes $225 s$ (top) to finish while the TDMA based system takes $194 s$ to finish (bottom).

host networking stack, we do not use a TCP stack in our experiments. Instead, raw Ethernet frames are transferred between the end hosts. The TDMA system employs an empirically chosen slot time of $300 \mu$ s (and guard times of $15 \mu \mathrm{s})$. The finish time of the overall transfer of the same size, 194 seconds, is about $15 \%$ better than the corresponding TCP finish time and only 5\% slower than ideal (due almost entirely to the guard band). The better finish time is achieved by ensuring that the flows are well coordinated and finish at the same time effectively using the available bandwidth.

\subsubsection{Multi-hop topology}

The non-blocking case is admittedly fairly trivial. Here, we consider spreading the 24 hosts across three separate switches ( 8 hosts per switch) and connect these three switches to a fourth aggregation switch. We implement this topology with two physical switches by using VLANs to create logical switches. We then perform the same set of experiments as before. The top and bottom portions of Figure 8 show the results for TCP and TDMA, respectively. The finish times are 1225 seconds for TCP and 1075 seconds for TDMA, compared to the optimal completion time of 1024 seconds. As we describe in Section 4.4 the controller has 3 NICs each of which is connected directly to the edge switches. This configuration allows us to send pause frames to the end hosts with the same precision that we achieve in the non-blocking scenario. We use the same TDMA slot settings as described previously, but our scheduler takes advantage of the higher bandwidth between two hosts on the same switch. Thus, the flows between hosts in the same 

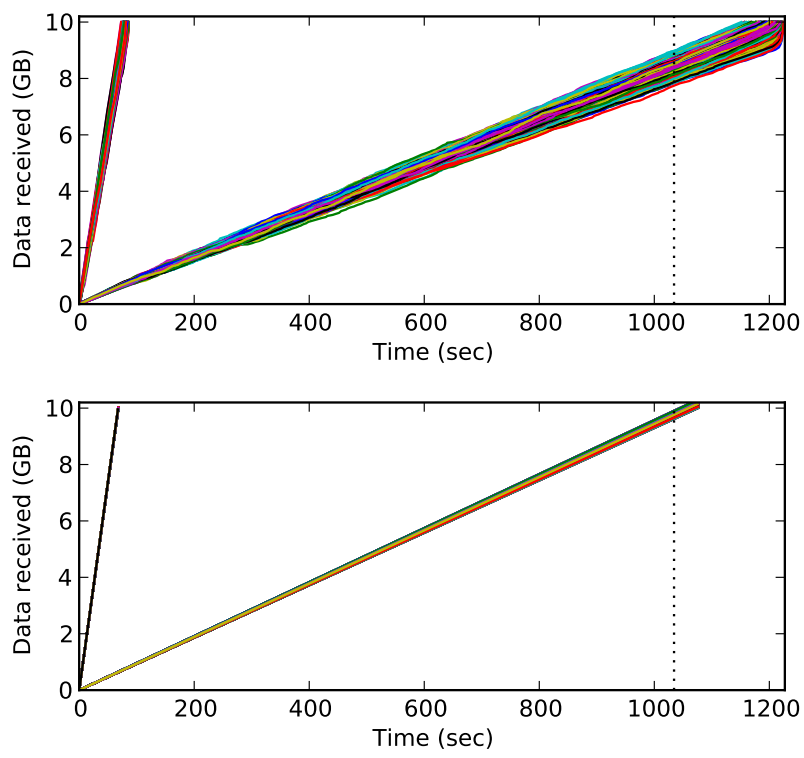

Figure 8. 10-GB all-to-all transfer between 24 hosts in a tree topology. TCP over regular Ethernet takes 1225s (top) to finish while the TDMA based system takes $1075 s$ to finish (bottom).

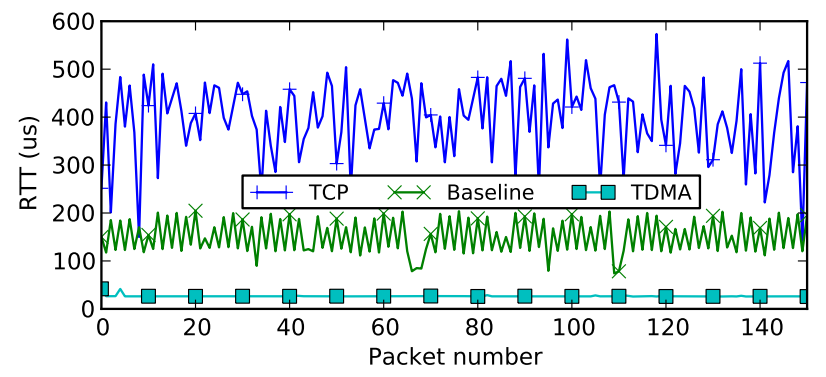

Figure 9. Hosts in the TDMA system have a 3x lower RTT than hosts in the presence of other TCP flows

switch finish earlier than the flows that go across switches, just as with TCP.

\subsection{Managing delay}

In a TDMA based system, the send times of hosts are controlled by a central manager. But, when the hosts get to send data they have unrestricted access to the network. This should mean that when a host has access to the channel it should experience very low latency to the destination host even in the presence of other large flows (that are assigned other slots). On the other hand, in a typical datacenter environment, TCP flows occupy the buffers in the switches increasing the latency for short flows-a key challenge facing applications that use the Partition/Aggregate model and require dependably low latency.

To illustrate this, we show that in the presence of longlived TCP flows the RTT between hosts increases. We use

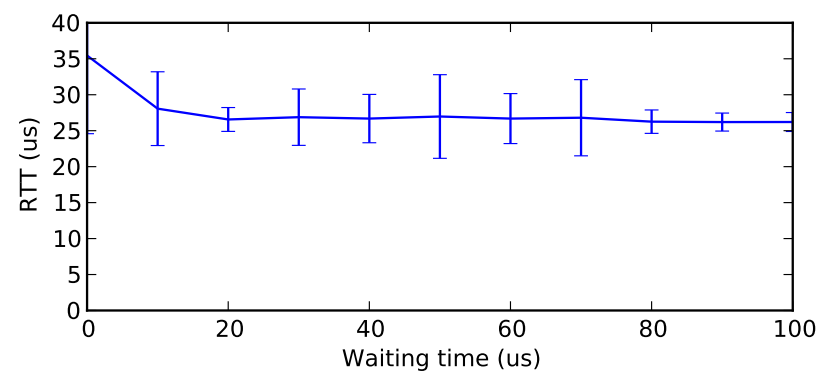

Figure 10. RTT between two hosts as a function of the time in the round when the ping is sent

the same 24-node, four-switch tree topology as before. We call the hosts connected to each of the edge switches a pod. A host each in pod 0 and pod 2 sends a long-lived TCP flow to a host in pod 1. While these flows are present we send a UDPbased ping from a host in pod 0 to a different host in pod 1 . The host which receives the ping immediately responds and we measure the RTT between the hosts. This RTT is shown with the TCP line in Figure 9. As expected, the RTT is high and variable due to the queue occupancy at the switch caused by the TCP cross traffic.

In contrast, in our TDMA-based system (where neither UDP nor TCP is employed) the switch buffers are empty during the slots assigned to the ping traffic resulting in a stable, low RTT. Since the host sending ping gets access to the channel for the entire slot, it can choose to send the ping at any time during the slot. Depending on when the ping is sent, it provides more time for the few buffers still in the switch to be emptied and hence achieve lower RTT. We show this in Figure 10. While we do not show it here due to lack of space, the average of $26 \mu$ s compares favorably with the RTT measurement in the absence of any traffic.

The reduced RTT is due to two factors, 1) usage of lowlevel kernel bypass at the end hosts and 2) near-zero buffer occupancy in the switches. To separate the two effectsas the former does not require TDMA-we measure RTT between the hosts in the testbed using UDP-based ping in the absence of other traffic and plot this as "baseline" in Figure 9. This shows that the TDMA system would achieve a 95th percentile RTT of $170 \mu$ s even without the kernel bypass, which is still over a $3 \times$ reduction.

The overhead of the TDMA approach is that when sending a ping, the hosts transmitting bulk data have to be de-scheduled and, hence, the long-lived flows could take longer to finish depending on the choice of schedule. For this experiment, we send a ping packet once every $30 \mathrm{~ms}$, that is, once every 100 TDMA slots. Thus, we see about a $1 \%$ hit in the transfer time of the large flows.

\subsection{Traffic oscillation}

The lack of coordination amongst TCP flows can have a large impact on the performance of a network. To illustrate this we run an experiment in which each host sends a fixed- 


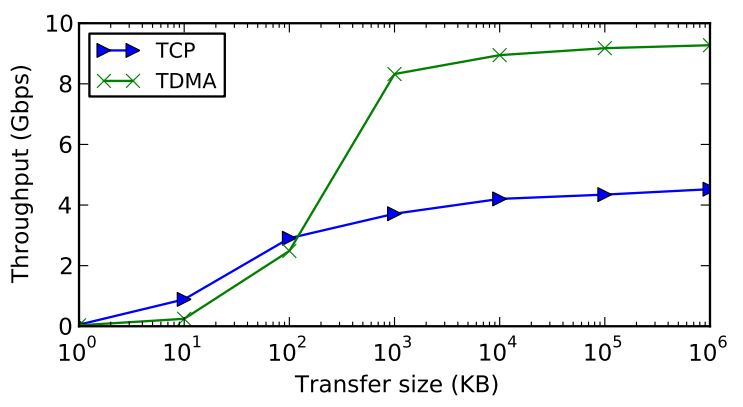

Figure 11. Throughput of the TCP system and TDMA system for round robin transfers with varying unit transfer size

sized block of data to a neighbor and once that transfer is finished, it moves on to the next neighbor. In the end we measure the average throughput achieved at each host. In a non-blocking scenario, if the hosts are perfectly synchronized then they should be able to communicate at link speed because at any point of time a link is used by a single flow.

Figure 11 shows that this is not the case with TCP regardless of block size. TCP achieves a best performance of about 4.5 Gbps on links which have 10 Gbps capacity. The TDMA-based system on the other hand can control the access to the links in a fine-grained manner and achieve higher throughput. The performance of our TDMA system begins to suffer as the unit transfer size desired is smaller than the amount of data that can be transferred in a TDMA slot (at 10 Gbps a $300-\mu$ s slot can accommodate $375 \mathrm{~KB}$ of data).

\subsection{Dynamic network configurations}

TCP is fundamentally reactive and takes a few RTTs to adapt to changes in the network. This can lead to very inefficient performance in scenarios where the link bandwidth fluctuates rapidly. A TDMA-based system, on the other, can avoid this through explicit scheduling. Here we evaluate the potential benefits of employing our TDMA underneath TCP in these environments using our pause-frame approach.

We emulate an extreme version of the optical/electrical link flapping scenario found in Helios [10] and c-through [26] by transferring data between two end hosts while a host between them acts as a switch. The host in the middle has two NICs, one connected to each of the other hosts. It forwards the packets that it receives on one NIC out the other NIC. We use the Myricom sniffer API which lets us receive the packet with very low latency in user space and send it out at varying rates. We oscillate the forwarding rate (link capacity) between $10 \mathrm{Gbps}$ and 1 Gbps every $10 \mathrm{~ms}$. The choice of oscillation interval is based upon an estimate of the time that TCP would take to adapt to the changing link capacities in the system: The RTT, including application reception and processing, is about $250 \mu \mathrm{s}$. Thus, TCP should take about $8 \mathrm{~ms}$ to ramp up from $1 \mathrm{Gbps}$ to $10 \mathrm{Gbps}$.

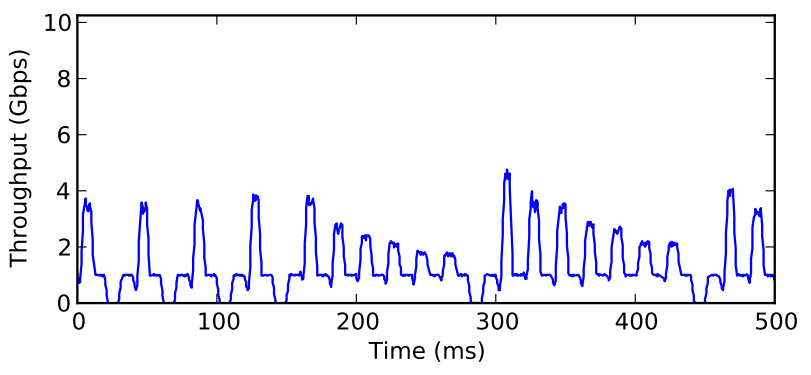

Figure 12. Bandwidth seen by the receiver in case of regular TCP adapting to changing link capacities.

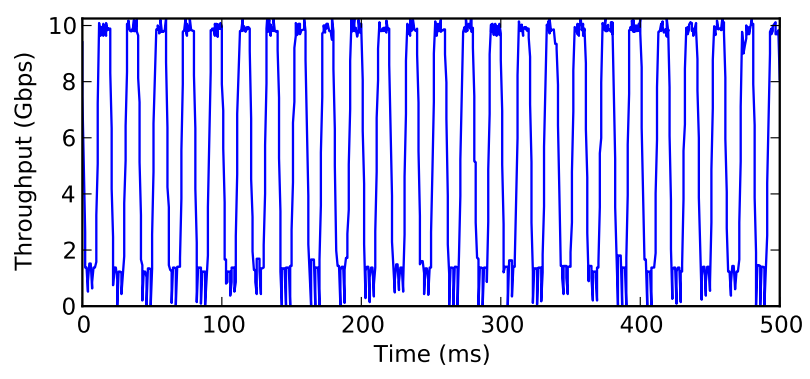

Figure 13. Bandwidth seen by the receiver when the TCP flow is controlled by TDMA.

Figure 12 shows the performance of TCP over regular Ethernet in the above scenario. Every $500 \mu$ s we plot the average bandwidth seen at the receiver over the preceding $2.5 \mathrm{~ms}$ for a period of $500 \mathrm{~ms}$. TCP does not ramp up quickly enough to realize the $10 \mathrm{Gbps}$ bandwidth before being throttled back to 1 Gbps. Moreover, TCP frequently drops to below 1 Gbps due to packet losses during the switch-over.

We can use our TDMA system to vary each end host's access to the channel. When the rate enforced is $10 \mathrm{Gbps}$ the host is scheduled every slot; it is scheduled only $\frac{1}{10}^{\text {th }}$ of the time when the rate being enforced is 1 Gbps. Figure 13 plots the performance of TCP when such rate limiting is done using $802.3 x$ pause frames. In this the host acting as the switch also functions as the fabric manager, scheduling the TCP flow using $802.3 x$ pause frames.

\subsection{Overhead of control traffic}

While the precise overhead of control traffic is dependent on the strategy used for demand collection from the end hosts, it is dominated by the pause frames sent by the fabric manager to end hosts-demand is signalled only once per round, but pause frames are sent per slot. We send two pause frames for each TDMA slot to each end host which is about $3 \mathrm{Mbps}$ per host. Half of this traffic (the pause frames that re-enable sending) is sent during the guard slot which means that the effective overhead of control traffic is about $1.5 \mathrm{Mbps}$ or $0.015 \%$ of the link capacity. 


\section{Conclusion and future work}

In this work, we propose to adapt an old approach to a new domain by deploying a TDMA-based MAC layer across an Ethernet datacenter network. Our approach, which does not require changes to network switches, relies on using link-layer flow control protocols to explicitly signal end hosts when to send packets. Our initial results show that the reduced in-network queuing and contention for buffer resources result in better performance for all-to-all transfer workloads and lower latency for request-response type workloads. Significant work remains, however, to evaluate how effectively a centralized scheduler can estimate demand and compute efficient slot assignments for real applications on arbitrary topologies. For example, we expect that some workloads-particularly those made up of unpredictable short flows, may be better serviced outside of the TDMA process. Moreover, while our system architecture should, in principle, allow the scheduler to react to switch, node, and link failures, we defer the evaluation of such a system to future work.

\section{Acknowledgments}

This work was funded in part by the National Science Foundation through grants CNS-0917339, CNS-0923523, and ERC-0812072. Portions of our experimental testbed were generously donated by Myricom and Cisco. We thank the anonymous reviewers and our shepherd, Jon Crowcroft, for their detailed feedback which helped us significantly improve the paper.

\section{References}

[1] J. A. Alegre, J. V. Sala, S. Péres, and J. Vila. RTL-TEP: An Ethernet protocol based on TDMA. In M. L. Chavez, editor, Fieldbus Systems and Their Applications 2005: Proceedings of the 6th IFAC International Conference, Nov. 2005.

[2] M. Alizadeh, A. Greenberg, D. A. Maltz, J. Padhye, P. Patel, B. Prabhakar, S. Sengupta, and M. Sridharan. Data center TCP (DCTCP). In ACM SIGCOMM, pages 63-74, Aug. 2010.

[3] A. Bavier, N. Feamster, M. Huang, L. Peterson, and J. Rexford. In VINI veritas: realistic and controlled network experimentation. ACM SIGCOMM, pages 3-14, Sept. 2006.

[4] H. H. Bazzaz, M. Tewari, G. Wang, G. Porter, T. S. E. Ng, D. G. Andersen, M. Kaminsky, M. A. Kozuch, and A. Vahdat. Switching the optical divide: Fundamental challenges for hybrid electrical/optical datacenter networks. In ACM SOCC, Oct. 2011.

[5] R. Braden, D. Clark, and S. Shenker. Integrated Services in the Internet Architecture: an Overview. RFC 1633, June 1994.

[6] F. Chang, J. Dean, S. Ghemawat, W. C. Hsieh, D. A. Wallach, M. Burrows, T. Chandra, A. Fikes, and R. E. Gruber. Bigtable: A Distributed Storage System for Structured Data. ACM Trans. Comput. Syst., 26:4:1-4:26, June 2008.

[7] Y. Chen, R. Griffith, J. Liu, R. H. Katz, and A. D. Joseph. Understanding TCP incast throughput collapse in datacenter networks. In WREN, pages 73-82, 2009.
[8] Y.-C. Cheng, J. Bellardo, P. Benkö, A. C. Snoeren, G. M. Voelker, and S. Savage. Jigsaw: Solving the puzzle of enterprise 802.11 analysis. In ACM SIGCOMM, Sept. 2006.

[9] J. Dean and S. Ghemawat. MapReduce: simplified data processing on large clusters. In USENIX OSDI, Dec. 2004.

[10] N. Farrington, G. Porter, S. Radhakrishnan, H. H. Bazzaz, V. Subramanya, Y. Fainman, G. Papen, and A. Vahdat. Helios: a hybrid electrical/optical switch architecture for modular data centers. In ACM SIGCOMM, pages 339-350, Aug. 2010.

[11] Juniper Networks. Opportunities and challenges with the convergence of data center networks. Technical report, 2011.

[12] T. Kohno, A. Brodio, and kc claffy. Remote Physical Device Fingerprinting. In Proceedings of the IEEE Symposium and Security and Privacy, Oakland, CA, May 2005.

[13] H. Kopetz, A. Ademaj, P. Grillinger, and K. Steinhammer. The time-triggered Ethernet (TTE) design. In IEEE Int'l Symp. on Object-oriented Real-time Distributed Comp., May 2005.

[14] T. Koponen, M. Casado, N. Gude, J. Stribling, L. Poutievski, M. Zhu, R. Ramanathan, Y. Iwata, H. Inoue, T. Hama, and S. Shenker. Onix: a distributed control platform for large-scale production networks. USENIX OSDI, Oct. 2010.

[15] Nistica, Inc. http://www. nistica. com/.

[16] D. Ongaro, S. M. Rumble, R. Stutsman, J. Ousterhout, and M. Rosenblum. Fast crash recovery in RAMCloud. In ACM SOSP, pages 29-41, Oct. 2012.

[17] OpenFlow. http://www. openflow.org.

[18] J. Ousterhout, P. Agrawal, D. Erickson, C. Kozyrakis, J. Leverich, D. Mazières, S. Mitra, A. Narayanan, G. Parulkar, M. Rosenblum, S. M. Rumble, E. Stratmann, and R. Stutsman. The case for RAMClouds: Scalable high-performance storage entirely in DRAM. ACM SIGOPS OSR, 43(4), Dec. 2009.

[19] P. Pedreiras, L. Almeida, and P. Gai. The FTT-Ethernet protocol: Merging flexibility, timeliness and efficiency. In Euromicro Conference on Real-Time Systems, June 2002.

[20] A. Phanishayee, E. Krevat, V. Vasudevan, D. G. Andersen, G. R. Ganger, G. A. Gibson, and S. Seshan. Measurement and analysis of TCP throughput collapse in cluster-based storage systems. USENIX FAST, Feb. 2008.

[21] A. Rasmussen, G. Porter, M. Conley, H. V. Madhyastha, R. N. Mysore, A. Pucher, and A. Vahdat. Tritonsort: A balanced large-scale sorting system. In USENIX NSDI, Mar. 2011.

[22] J. Rothschild. High performance at massive scale lessons learned at facebook. http://video-jsoe.ucsd.edu/ asx/JeffRothschildFacebook . asx, Oct. 2009.

[23] R. Santos, A. Vieira, P. Pedreiras, A. Oliveira, L. Almeida, R. Marau, and T. Nolte. Flexible, efficient and robust realtime communication with server-based Ethernet switching. In IEEE Workshop on Factory Comm. Systems, May 2010.

[24] A. Singla, A. Singh, K. Ramachandran, L. Xu, and Y. Zhang. Proteus: a topology malleable data center network. In $A C M$ HotNets, Oct. 2010.

[25] V. Vasudevan, A. Phanishayee, H. Shah, E. Krevat, D. G. Andersen, G. R. Ganger, G. A. Gibson, and B. Mueller. Safe and effective fine-grained TCP retransmissions for datacenter 
communication. In ACM SIGCOMM, pages 303-314, Aug. 2009.

[26] G. Wang, D. G. Andersen, M. Kaminsky, K. Papagiannaki, T. E. Ng, M. Kozuch, and M. Ryan. c-Through: part-time optics in data centers. In ACM SIGCOMM, Aug. 2010.

[27] K. Webb, A. C. Snoeren, and K. Yocum. Topology switching for data center networks. In USENIX Hot-ICE, Mar. 2011.
[28] H. Wu, Z. Feng, C. Guo, and Y. Zhang. ICTCP: Incast congestion control for TCP in data center networks. In ACM CoNEXT, Dec. 2010.

[29] Scaling Hadoop to 4000 nodes at Yahoo! http: //developer.yahoo.net/blogs/hadoop/2008/ 09/scaling_hadoop_to_4000_nodes_a.html.

[30] L. Zhang, S. Deering, D. Estrin, S. Shenker, and D. Zappala. RSVP: A new resource reservation protocol. IEEE Communications, 40(5):116-127, May 2002. 